EPJ manuscript No.

(will be inserted by the editor)

\title{
Statistical properties of volatility return intervals of Chinese stocks
}

\author{
Fei Ren ${ }^{1,2, a}$, Liang Guo ${ }^{1}$, and Wei-Xing Zhou ${ }^{1,2,3,4, b}$ \\ 1 School of Business, East China University of Science and Technology, Shanghai 200237, China \\ 2 Research Center for Econophysics, East China University of Science and Technology, Shanghai 200237, China \\ 3 School of Science, East China University of Science and Technology, Shanghai 200237, China \\ ${ }^{4}$ Research Center of Systems Engineering, East China University of Science and Technology, Shanghai 200237, China
}

Received October 24, 2018/ Revised version:

\begin{abstract}
The statistical properties of the return intervals $\tau_{q}$ between successive 1-min volatilities of 30 liquid Chinese stocks exceeding a certain threshold $q$ are carefully studied. The Kolmogorov-Smirnov (KS) test shows that 12 stocks exhibit scaling behaviors in the distributions of $\tau_{q}$ for different thresholds $q$. Furthermore, the KS test and weighted KS test shows that the scaled return interval distributions of 6 stocks (out of the 12 stocks) can be nicely fitted by a stretched exponential function $f(\tau / \bar{\tau}) \sim e^{-\alpha(\tau / \bar{\tau})^{\gamma}}$ with $\gamma \approx 0.31$ under the significance level of 5\%, where $\bar{\tau}$ is the mean return interval. The investigation of the conditional probability distribution $P_{q}\left(\tau \mid \tau_{0}\right)$ and the mean conditional return interval $\left\langle\tau \mid \tau_{0}\right\rangle$ demonstrates the existence of short-term correlation between successive return interval intervals. We further study the mean return interval $\left\langle\tau \mid \tau_{0}\right\rangle$ after a cluster of $n$ intervals and the fluctuation $F(l)$ using detrended fluctuation analysis and find that long-term memory also exists in the volatility return intervals.
\end{abstract}

PACS. 89.65.Gh Economics; econophysics, financial markets, business and management - 89.75.Da Systems obeying scaling laws - 05.45.Tp Time series analysis

\section{Introduction}

In recent years many concepts and methods from statistical physics have been applied to the study of financial markets [1]. The statistical analysis of the waiting time between two successive events has drawn much attention. Different definitions of event refer to a variety of variables characterizing the properties of stock markets from certain aspects. For example, the persistence probability concerns the duration time that prices (or volatilities) always keep above or below their initial values [2, 3, 4], the exit time raises a concept similar to the persistence probability and focuses on the interval time between prices with fluctuations larger than a certain threshold [5,6,7, 8, 9, 10], and the intertrade duration stands for the time interval between two successive independent trades [11, 12, 13, 14].

Recently, extensive work has been done in the analysis of waiting time between extreme events in natural records, such as floods, temperatures and earthquakes [15,16,17,18]. The understanding of the statistical properties of these extreme events is of great importance for the risk assessment of rare events. By investigating the return intervals between successive extreme events exceeding a certain threshold $q$, scaling behaviors are revealed in the return interval distributions for numerous complex systems. The dependence of the return interval distribution on the correlation of the original records has been carefully in-

\footnotetext{
a e-mail: fren@ecust.edu.cn

b e-mail: wxzhou@ecust.edu.cn
}

vestigated [19,20,21,22,23]. It shows that the scaling behavior of the return intervals might be due to the long-term memory of the original records. It is well known that long memory is an important statistical property basically observed in stock volatilities [1]. Therefore, one can expect that the scaling behavior might also appear in the volatility records of stocks.

A first effort was conducted by Yamasaki et al., who used the daily data of US stocks to study the properties of the volatility return intervals [24]. Suppose that $Y(t)$ is the price at time $t$, and the volatility is simply defined as the magnitude of logarithmic return $R(t, \delta t)=|\ln (Y(t))-\ln (Y(t-\delta t))|$. They studied the return intervals $\tau$ between successive volatilities above a certain threshold $q$, and found that the distribution of volatility return intervals $P_{q}(\tau)$ indeed obeys a scaling behavior. Wang et al. further studied the statistical properties of the volatility return intervals using the intraday data of US stocks [25,26,27]. They found that $P_{q}(\tau)$ exhibited a similar scaling behavior and the distribution could be approximated by a stretched exponential form similar to that found in climate records [17]. All these studies show that the volatility return intervals have long memory. Similar scaling behavior and longterm memory are observed in the daily and 1-min volatility return intervals of thousands Japanese stocks [28]. Qiu et al. studied the volatility return intervals using the high-frequency intraday data of four liquid stocks traded in the Chinese market, which is an important emerging market, and found the return interval distribution also follows scaling behavior [29]. 
Fei Ren et al.: Statistical properties in the volatility return intervals of Chinese stocks

Contrary to the scaling behavior found previously, Wang et al. analyzed the trade-by-trade data of 500 stocks which compose the S\&P 500 index and found that the cumulative distribution of return intervals had systematic deviations from scaling and showed multiscaling behaviors [30]. The analysis was based on the deduction that if the probability distribution obeys scaling behavior its cumulative distribution should also obeys scaling behavior. They further studied the $m$-th moment of the scaled return intervals, and found the moment showed a certain trend with the mean interval which supports the finding that the return interval distribution exhibits a multiscaling behavior. This remarkable multiscaling behavior calls for a more careful scrutiny of the volatility return intervals by adopting solid statistical methods rather than eyeballing. In addition, Lee et al. investigated the return intervals of 1-min volatility data of the Korean KOSPI index [31]. They found that the interval return distribution had a power-law tail and no scaling was observed. However, it seems that they did not remove the intraday pattern from the intraday volatility series, which weakens their conclusion. Note that intraday pattern should be removed when dealing with intraday data, which will have heavy influence on the estimated return intervals for large thresholds.

In this work, we use a nice high-frequency database [32] to study the statistical properties of the interval returns. The two samples Kolmogorov-Smirnov (KS) test, which is a standard way to check whether these two samples are drawn from a same distribution by comparing their cumulative distributions, is used to examine the coincidence of the interval distributions for different values of threshold $q$. Specifically, we study in detail 30 most liquid stocks to gain better statistics. Our results show that only 12 individual stocks pass the KS test and show scaling behaviors, and the remaining 18 stocks show nonscaling behaviors which seems consistent with that found by Wang et al. [30]. Furthermore, a KS goodness of fit test is performed to study the particular form of the scaling function. The memory effect is also investigated in this paper.

The paper is organized as follows. In Section 2, we explain the database analyzed and how the volatility return intervals are calculated. Section 3 studies the scaling and nonscaling behaviors of the volatility return interval distributions using the KS tests. In Section 4, we further study the memory effect of volatility return intervals. Section 5 concludes.

\section{Preprosessing the data sets}

Our analysis is based on the high-frequency intraday data of 30 most liquid stocks traded on the Shanghai Stock Exchange and the Shenzhen Stock Exchange. These 30 stocks are most actively traded stocks representative in a variety of industry sectors, and thus have the largest sizes among all the stocks. The basic information about these stocks including the stock codes, company names and industrial sectors they belonging to is shown in Table 1 The dada record the trading prices every six to eight seconds from January 2004 to June 2006. The volatility is defined as the magnitude of logarithmic price return between two consecutive minutes, that is $R(t)=\mid \ln (Y(t))-$ $\ln (Y(t-1)) \mid$, where the price is the closest tick to a minute mark. Thus the sampling time is one minute, and the volatility data size is about 140,000 for each stock.
For most stock markets, the intraday volatilities exhibit a U-shaped or L-shaped intraday pattern. This is so for the Chinese stocks [33]. When dealing with intraday data, this pattern should be removed [25, 26, 27, 30,29]. Otherwise, the return intervals distribution will exhibit daily periodicity for large thresholds. The intraday pattern $A(s)$ is defined as

$$
A(s)=\frac{\sum_{i=1}^{N} R^{i}(s)}{N}
$$

which is the volatility at a specific moment $s$ of the trading day averaged over all $N$ trading days and $R^{i}(s)$ is the volatility at time $s$ of day $i$. The L-shaped intraday patterns of four typical stocks are illustrated in Figure 1. The intraday pattern exhibits a pronounced peak at the opening hours, and a minimum value just before the midday break of a trading day. In comparison with the western stock markets, the intraday pattern of the Chinese stock market surges soon after midday break which may due to the information aggregation during the midday break.

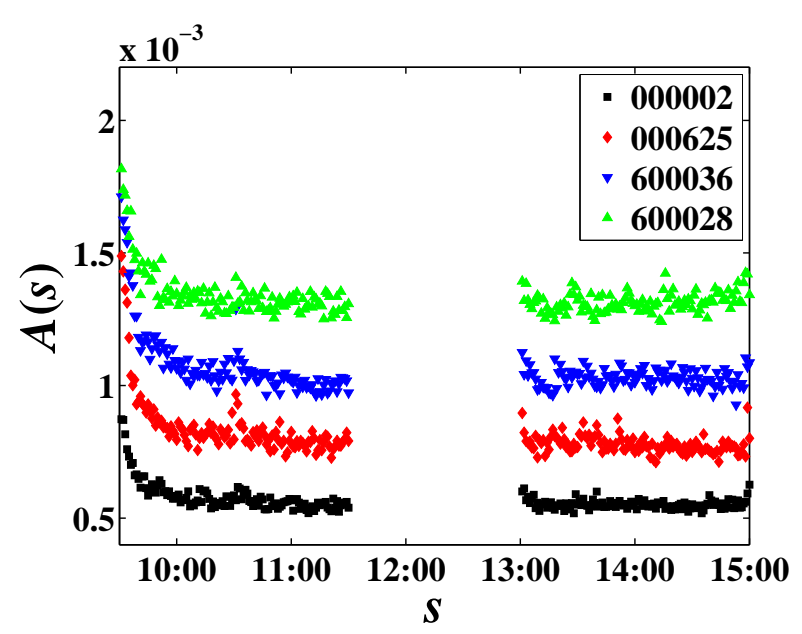

Fig. 1. (Color online) The 1-min interval intraday patterns of four representative stocks: 000002, 000625, 600036 and 600028. The curves are vertically shifted for clarity.

To avoid the effect of this daily oscillation, the intraday pattern is removed by

$$
R^{\prime}(t)=\frac{R(t)}{A(s)} .
$$

Then we normalize the volatility by dividing its standard deviation

$$
r(t)=\frac{R^{\prime}(t)}{\left[\left\langle R^{\prime}(t)^{2}\right\rangle-\left\langle R^{\prime}(t)\right\rangle^{2}\right]^{1 / 2}} .
$$

We note that the raw 1-min Chinese stock returns follow Student distribution whose tail exponent is about 3.5 [34], which ensures that the variance of $R^{\prime}(t)$ exists where the intraday pattern is removed.

We study the return intervals $\tau$ between successive volatilities $|r|$ exceeding a certain threshold $q$. The definition of return intervals is illustrated in Figure 2 for a typical stock. It is clear that the number of return intervals corresponding to a given threshold decreases with increasing threshold and the sum of 
Fei Ren et al:: Statistical properties in the volatility return intervals of Chinese stocks

Table 1. Kolmogorov-Smirnov test of the return interval distributions for $q=2$ and 5 by comparing the statistic $K S$ with the critical value $C V$ at the $5 \%$ significance level.

\begin{tabular}{|c|c|c|c|c|c|}
\hline Code & Stock name & Industrial sector & $K S$ & $C V$ & Scaling? \\
\hline 000002 & China Vanke Co., Ltd & Real estate & 0.0793 & 0.0636 & No \\
\hline 000503 & Searainbow Holding Co., Ltd & Conglomerates & 0.0485 & 0.0469 & No \\
\hline 000625 & Chongqing Changan Automobile Co., Ltd & Manufacturing & 0.0233 & 0.0509 & Yes \\
\hline 000839 & Citic Guoan Information Industry Co., Ltd & IT & 0.0705 & 0.0490 & No \\
\hline 000858 & Wuliangye Yibin Co., Ltd & Wine & 0.0803 & 0.0500 & No \\
\hline 000917 & Hunan TV\&Broadcast Intermediary Co., Ltd & Broadcasting & 0.0718 & 0.0511 & No \\
\hline 000930 & Anhui BBCA Biochemical Co., Ltd & Biology & 0.0699 & 0.0518 & No \\
\hline 000983 & Shanxi Xishan Coal and Electricity Power Co., Ltd & Power electronics & 0.0509 & 0.0496 & No \\
\hline 600000 & Shanghai Pudong Development Bank Co., Ltd & Financials & 0.0699 & 0.0538 & No \\
\hline 600019 & Baoshan Iron \& Steel CO., LTD & Metals & 0.0223 & 0.0672 & Yes \\
\hline 600026 & China Shipping Development Co., Ltd & Transportation & 0.0277 & 0.0511 & Yes \\
\hline 600028 & China Petroleum \& Chemical Co., Ltd & Petroleum \& Chemistry & 0.0670 & 0.0653 & No \\
\hline 600030 & CITIC Securities Company Co., Ltd & Financials & 0.0580 & 0.0520 & No \\
\hline 600036 & China Merchants Bank Co., Ltd & Financials & 0.0436 & 0.0576 & Yes \\
\hline 600073 & Shanghai Maling Aquarius Co., Ltd & Food & 0.0501 & 0.0500 & No \\
\hline 600088 & China Television Media Co., Ltd & Broadcasting & 0.0540 & 0.0497 & No \\
\hline 600100 & Tsinghua Tongfang Co., Ltd & IT & 0.0462 & 0.0500 & Yes \\
\hline 600104 & SAIC Motor CO., LTD & Manufacturing & 0.0149 & 0.0573 & Yes \\
\hline 600110 & China-Kinwa High Technology Co., Ltd & Manufacturing & 0.0911 & 0.0506 & No \\
\hline 600171 & Shanghai Belling Co., Ltd & Electronics & 0.0377 & 0.0530 & Yes \\
\hline 600320 & Shanghai Zhenhua Port Machinery Co., Ltd & Manufacturing & 0.0515 & 0.0506 & No \\
\hline 600428 & COSCO Shipping Co., Ltd & Transportation & 0.0475 & 0.0510 & Yes \\
\hline 600550 & Baoding Tianwei Baobian Electric Co., Ltd & Manufacturing & 0.0198 & 0.0498 & Yes \\
\hline 600601 & Founder Technology Group Co., Ltd & IT & 0.0218 & 0.0637 & Yes \\
\hline 600602 & SVA Electron Co., Ltd & Electronics & 0.0167 & 0.0561 & Yes \\
\hline 600688 & SINOPEC Shanghai Petrochemicl Co., Ltd & Petroleum \& Chemistry & 0.0421 & 0.0566 & Yes \\
\hline 600770 & Jiangsu Zongyi Co., Ltd & Conglomerates & 0.0647 & 0.0478 & No \\
\hline 600797 & INSIGMA Technology Co., Ltd & IT & 0.1179 & 0.0647 & No \\
\hline 600832 & Shanghai Oriental Pearl (Group) Co.Ltd & Conglomerates & 0.0610 & 0.0474 & No \\
\hline 600900 & China Yangtze Power Co., Ltd & Power electronics & 0.0574 & 0.0556 & No \\
\hline
\end{tabular}

return intervals $\tau_{q}$ are approximately identical to each other for different $q$. When the threshold $q$ is very small, say less than the minimum of $r(t)$, then all $\tau_{q}$ values equal to 1 .

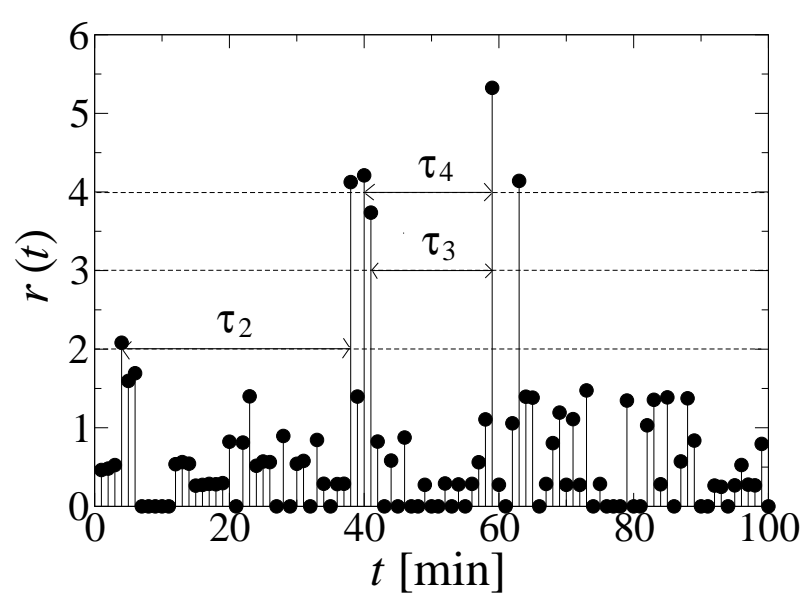

Fig. 2. Illustration of volatility return intervals for stock 000625 , where $\tau_{2}, \tau_{3}$ and $\tau_{4}$ correspond to return intervals for $q=2,3$ and 4 .

\section{Scaling and nonscaling behaviors of return interval distributions}

Several empirical studies show that the probability distribution function (PDF) of the return intervals obeys a scaling form:

$$
P_{q}(\tau)=\frac{1}{\bar{\tau}} f\left(\frac{\tau}{\bar{\tau}}\right)
$$

where $\bar{\tau}$ is the mean return interval which depends on the threshold $q$. The scaling form could be approximated by a stretched exponential function as

$$
f(x)=c e^{-\alpha x^{\gamma}},
$$

where $c$ and $\alpha$ are two parameters and $\gamma$ is the correlation exponent characterizing the long-term memory of volatilities. In this section, we investigate if these two properties hold for the 30 Chinese stocks.

\subsection{Complementary cumulative distributions}

To make the observation of this possible scaling behavior (4) more clear, we study the complementary cumulative distribution function (CCDF) of the scaled return intervals

$$
C_{q}(\tau / \bar{\tau})=\int_{\tau}^{\infty} P_{q}(\tau) d \tau=\int_{\tau / \bar{\tau}}^{\infty} f(x) d x .
$$


Fei Ren et al.: Statistical properties in the volatility return intervals of Chinese stocks
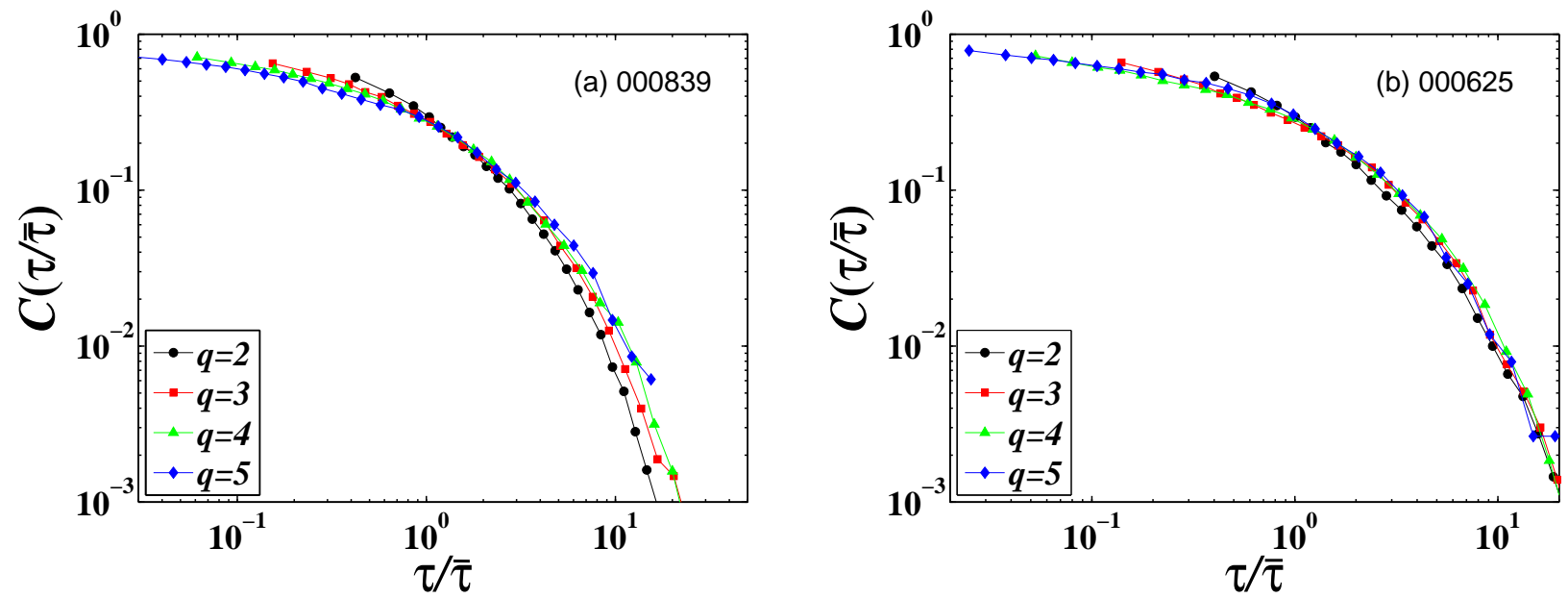

Fig. 3. (Color online) Empirical complementary cumulative distribution of scaled return intervals for different thresholds $q=2,3,4,5$ for (a) stock 000839 which shows clear deviations from scaling, and (b) stock 000625 which apparently shows scaling behavior.

If the PDFs for different $q$ obey the scaling form in Eq. (4), the CCDFs of the scaled return intervals should also collapse onto a single curve.

We calculate the CCDFs of the scaled return intervals of the chosen 30 stocks for a wide range of threshold $q=2,3,4,5$. Note that $q$ is in units of standard deviations such that $q=5$ is a quite large volatility. The CCDFs of a representative stock 000839 are shown in Figure 3 a). The curves for different values of threshold $q$ seem close to each other but do not collapse onto a single curve. A systematic deviation from scaling is clearly observed: the cumulative distribution decreases with the increase of threshold $q$ for small scaled return intervals $\tau / \bar{\tau}<1$, while it increases with $q$ for large scaled return intervals $\tau / \bar{\tau}>1$. This observation is similar to the multiscaling behavior reported by Wang et al. [30].

However, we also find some stocks which apparently show scaling behaviors. In Figure 3 b), the CCDFs of a representative stock 000625 that exhibits approximate scaling behavior are plotted. One observes that the curves for different values of threshold $q$ approximately collapse onto a single curve. This indicates the return interval distributions of this stock obey a scaling behavior.

\subsection{Kolmogorov-Smirnov test of scaling in return interval distributions}

The eyeballing of complementary cumulative distributions offers a qualitative way of distinguishing scaling and nonscaling behaviors. Here we further adopt a quantitative approach, the Kolmogorov-Smirnov (KS) test. We use the KS test to compare two distributions for $q=2$ and $q=5$, which behave most differently among all the $q$ value distributions. If the distributions for $q=2$ and $q=5$ pass the test, we can conclude that all the return interval distributions for different $q$ values collapse onto a single curve and thus obey a scaling law.

The standard KS test is designed to test the hypothesis that the distribution of the empirical data is equal to a particular distribution by comparing their cumulative distribution functions
(CDFs). Our hypothesis is that the two return interval distributions for $q=2$ and $q=5$ do not differ at least in the common region of the scaled return intervals [28]. Suppose that $F_{2}=1-C_{2}$ is the CDF of return intervals for $q=2$ and $F_{5}=1-C_{5}$ is the CDF of return intervals for $q=5$. We calculate the KS statistic by comparing the two CDFs in the overlapping region:

$$
K S=\max \left(\left|F_{2}-F_{5}\right|\right) .
$$

When the KS statistic is smaller than a critical value denoted by $C V$ (i.e., $K S<C V$ ), the hypothesis is accepted and we can assume that the distribution for $q=2$ is coincident with the distribution for $q=5$. The critical value at the significance level of 5\% is $C V=1.36 / \sqrt{m n /(m+n)}$, where $m$ and $n$ are the numbers of interval samples for $q=2$ and 5 , respectively. In Table 1 is depicted the KS statistics and the corresponding critical values for the 30 stocks. One can see that 12 stocks including the stock shown in Figure 3(b) pass the KS test and consequently their return interval distributions obey scaling behaviors, while other 18 stocks including the one shown in Figure 3 a) do not pass the test. We find that stock 600030, which is the only stock also studied by Qiu et al. [29], does not pass the KS test at the significance level of 5\%, but the statistic $K S$ is very close to the critical value $C V$, i.e., $0.0580 \sim 0.0520$. Further study shows that the stock 600030 passes the KS test at a significance level of $1.97 \%$ which indicates this stock may follow a relatively week scaling behavior.

\subsection{Kolmogorov-Smirnov test of the scaling function}

We have demonstrated that the return distributions of 12 stocks show scaling behaviors. To further study the particular form of the scaling function, we perform the KS goodness-of-fit test [35,36]. Empirical studies have shown that the scaling form could be approximated by a stretched exponential function as in Eq. (5). In this case, our hypothesis is that the empirical distribution is coincident with the fitted stretched exponential distribution. Similar to the KS test we have conducted for two 
Fei Ren et al.: Statistical properties in the volatility return intervals of Chinese stocks

empirical samples, we use the KS statistics to test whether the distributions for $q=2$ and $q=5$, which behave most differently among all the $q$ value distributions, are identical to a same fitted distribution for both $q$ values in the overlapping region of the scaled return intervals. If both return interval distributions for $q=2$ and $q=5$ pass the test, we can conclude that all the return interval distributions for different $q$ values follow a scaling function with stretched exponential form.

The method is described as follows. Let $F_{q}=1-C_{q}$ be the cumulative distribution of $\tau_{q}, F_{S E}$ the cumulative distribution from the fitted stretched exponential. The KS statistic defined in Eq. (7) becomes

$$
K S=\max \left(\left|F_{q}-F_{\mathrm{SE}}\right|\right), \quad q=2,5 .
$$

We also use an variant of the KS statistic known as the weighted KS statistic, which is defined as follows [36]

$$
K S W=\max \left(\frac{\left|F_{q}-F_{\mathrm{SE}}\right|}{\sqrt{F_{\mathrm{SE}}\left(1-F_{\mathrm{SE}}\right)}}\right),
$$

which is more sensitive on the edges of the cumulative distribution. In this case, the bootstrapping approach is adopted [35. 36]. To do this, we first generate 1000 synthetic samples from the best fitted distribution and then reconstruct the cumulative distribution $F_{\text {sim }}$ of each simulated sample and its CDF $F_{\text {sim, SE }}$ from integrating the fitted stretched exponential. We calculate the values of $K S$ and $K S W$ between the fitted CDF and the simulated CDF using

$$
K S_{\mathrm{sim}}=\max \left(\left|F_{\mathrm{sim}}-F_{\mathrm{sim}, \mathrm{SE}}\right|\right)
$$

and

$$
K S W_{\mathrm{sim}}=\max \left(\frac{\left|F_{\mathrm{sim}}-F_{\mathrm{sim}, \mathrm{SE}}\right|}{\sqrt{F_{\mathrm{sim}, \mathrm{SE}}\left(1-F_{\mathrm{sim}, \mathrm{SE}}\right)}}\right) .
$$

The $p$-value is determined by the frequency that $K S_{\text {sim }}>K S$ or $K S W_{\text {sim }}>K S W$. The tests are carried out for the 12 stocks exhibiting scaling behaviors. The resultant $p$-values are depicted in Table 2 .

Consider the significance level of $1 \%$. If at least one $p$ value for $q=2$ or $q=5$ of an individual stock is less than $1 \%$, then the null hypothesis that the empirical PDFs of this stock can be well fitted by a stretched exponential is rejected. According to Table 2, the null hypothesis is rejected for three stocks $(600100,600428,600601)$ using the KS test and for two stocks $(600428,600601)$ using the KSW test. Under the significant level of $5 \%$, the null hypothesis is rejected for six stocks $(000625,600026,600100,600428,600601,600688)$ using the KS test and for five stocks (000625, 600026, 600100, 600428, $600601)$ using the KSW test. We find that the KS test and the KSW test provide very similar results except that the KS test is slightly stronger [36]. It is noteworthy to point out that the $p$-values of stocks 600602, 600019 and 600104 are very large, implying high goodness-of-fit of the stretched exponential to the empirical PDFs. In principle, the $p$-values of a stock are larger when the scaling of PDFs for different $q$ is more significant, which is manifested by the fact that the statistic $K S$ exceeds the critical value $C V$ more in Table 1
Table 2. $K S$ and $K S W$ test of the return interval distributions for $q=$ 2 and 5 by comparing empirical data with the best fitted distribution and synthetic data with the best fitted distribution.

\begin{tabular}{cccccccc}
\hline Code & $q$ & $p_{K S}$ & $p_{K S W}$ & Code & $q$ & $p_{K S}$ & $p_{K S W}$ \\
\hline 000625 & 2 & 0.224 & 0.769 & 600019 & 2 & 0.938 & 0.742 \\
& 5 & 0.045 & 0.023 & & 5 & 0.567 & 0.574 \\
600026 & 2 & 0.010 & 0.215 & 600036 & 2 & 0.311 & 0.432 \\
& 5 & 0.021 & 0.011 & & 5 & 0.086 & 0.098 \\
600100 & 2 & 0.003 & 0.022 & 600104 & 2 & 0.851 & 0.973 \\
& 5 & 0.157 & 0.184 & & 5 & 0.709 & 0.866 \\
600171 & 2 & 0.158 & 0.286 & 600428 & 2 & 0.004 & 0.001 \\
& 5 & 0.401 & 0.346 & & 5 & 0.002 & 0.007 \\
600550 & 2 & 0.226 & 0.354 & 600601 & 2 & 0 & 0 \\
& 5 & 0.059 & 0.103 & & 5 & 0.883 & 0.130 \\
600602 & 2 & 0.995 & 0.943 & 600688 & 2 & 0.038 & 0.386 \\
& 5 & 0.996 & 0.592 & & 5 & 0.188 & 0.056 \\
\hline
\end{tabular}

In all the fitting, the correlation exponent $\gamma$ for different stocks lies in a range $[0.22,0.44]$ with an average value 0.31 , which is consistent with that observed in [37]. To show how good the stretched exponential fits the data, we illustrate in Figure 4 the empirical PDFs of $\tau_{q}$ and the fitted curve for a representative stock 000625, which passes the KS and KSW goodness-of-fit tests at the significance level of $1 \%$ but falls at the significance level of 5\%. It is obviously that the curves for different values of $q$ almost collapse onto a single curve which could be nicely fitted by a stretched exponential function.

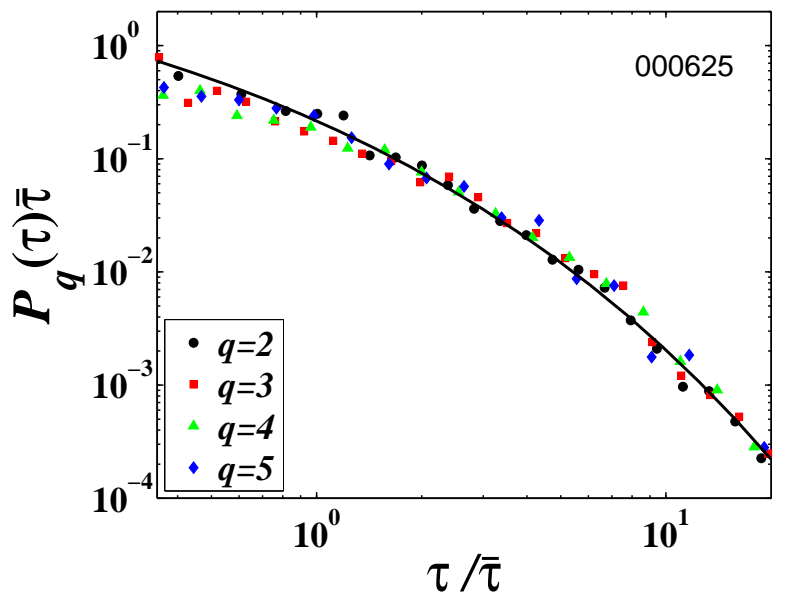

Fig. 4. (Color online) Scaled probability distribution of scaled return intervals for different threshold $q=2,3,4,5$ for the representative stock 000625 , which show scaling behavior. The solid curve is the fitted function $c e^{-\alpha x^{\gamma}}$.

\section{Memory effect of return intervals}

The probability distribution may not fully characterize the properties of volatility return intervals. The temporal correlation is known as another important observable independent of the 
probability distribution. Empirical studies have revealed that the stock market volatilities are long-term correlated. We suppose that the long-term correlated volatilities would also affect the memory of the return intervals. Indeed, the memory effect is observed in the return intervals of US stock market and Japanese stock market.

\subsection{Short-term memory of return intervals}

To investigate the memory effect of the return intervals in Chinese stock market, we first calculate the conditional probability distribution $P_{q}\left(\tau \mid \tau_{0}\right)$, which is the probability to find an interval $\tau$ immediately after the interval $\tau_{0}$. Specifically, we study the conditional PDF for a bin of $\tau_{0}$. The entire interval sequences are arranged in ascending order and partitioned to four bins with equal size. We illustrate the results using a typical stock 000002 . The results for all other 29 stocks are qualitatively the same. Figure 5 plots $P_{q}\left(\tau \mid \tau_{0}\right)$ as a function of the scaled return intervals $\tau / \bar{\tau}$ for $\tau_{0}$ in the smallest and biggest bins. For large $\tau / \bar{\tau}, P_{q}\left(\tau \mid \tau_{0}\right)$ with $\tau_{0}$ in the biggest subset is larger than $P_{q}\left(\tau \mid \tau_{0}\right)$ with $\tau_{0}$ in the smallest subset, and for small $\tau / \bar{\tau}, P_{q}\left(\tau \mid \tau_{0}\right)$ with $\tau_{0}$ in the biggest subset is smaller than $P_{q}\left(\tau \mid \tau_{0}\right)$ with $\tau_{0}$ in the smallest subset. This indicates that small intervals $\tau_{0}$ tend to be followed by small intervals $\tau$, while large intervals $\tau_{0}$ tend to be followed by large intervals $\tau$. Therefore, there exists short-term memory in the return intervals.

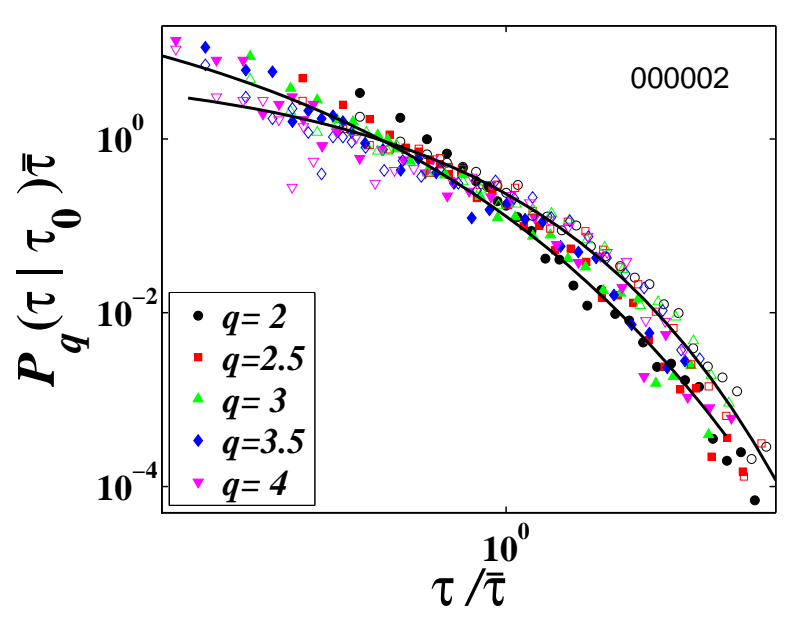

Fig. 5. (Color online) Scaled conditional PDF $P_{q}\left(\tau \mid \tau_{0}\right) \bar{\tau}$ of scaled return intervals $\tau / \bar{\tau}$ with $\tau_{0}$ in the largest $1 / 4$ subset (open symbols) and smallest $1 / 4$ subset (filled symbols) for a representative stock 000002. The solid lines are fitted curves.

Calculating the mean conditional return interval $\left\langle\tau \mid \tau_{0}\right\rangle$ immediately after $\tau_{0}$ is another alternative way to investigate the memory of return intervals. In fact, $\left\langle\tau \mid \tau_{0}\right\rangle$ is the first moment of $P_{q}\left(\tau \mid \tau_{0}\right)$. In Figure 6 is shown $\left\langle\tau \mid \tau_{0}\right\rangle$ of stock 000002, which exhibits a monotonously increasing tendency with the increase of the scaled return interval $\tau / \bar{\tau}$. This also indicates that small $\tau$ tends to follow small $\tau_{0}$ and big $\tau$ tends to follow big $\tau_{0}$. This result is consistent with that observed in $P_{q}\left(\tau \mid \tau_{0}\right)$. For those shuffled return intervals, $\left\langle\tau \mid \tau_{0}\right\rangle$ fluctuates around a horizontal line close to 1 indicating that $\tau$ is independent of the previous $\tau_{0}$, as expected. This suggests that the memory of the return intervals may arise from the long-term memory of the volatilities.

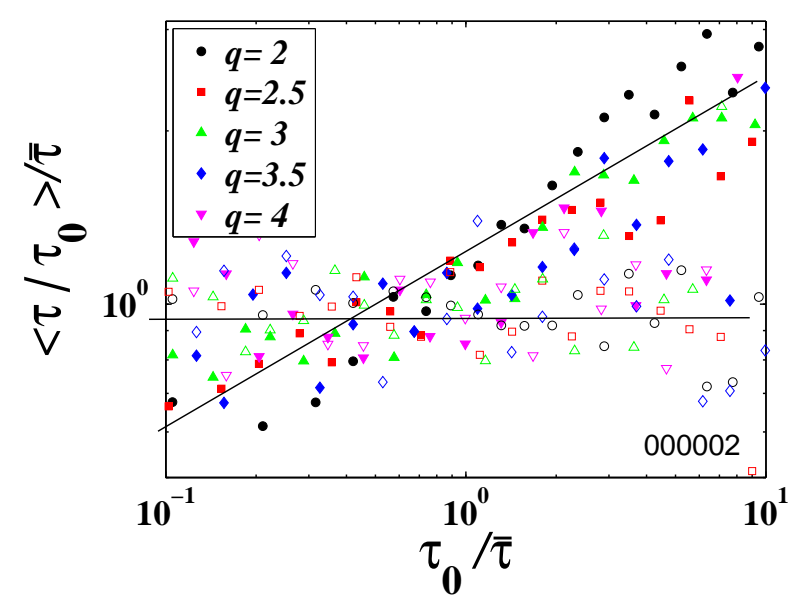

Fig. 6. (Color online) Scaled mean conditional return interval $\left\langle\tau \mid \tau_{0}\right\rangle / \bar{\tau}$ of scaled return intervals (filled symbols) and shuffled data (open symbols) for a typical stock 000002 . The solid lines are guidelines.

\subsection{Long-term memory of return intervals}

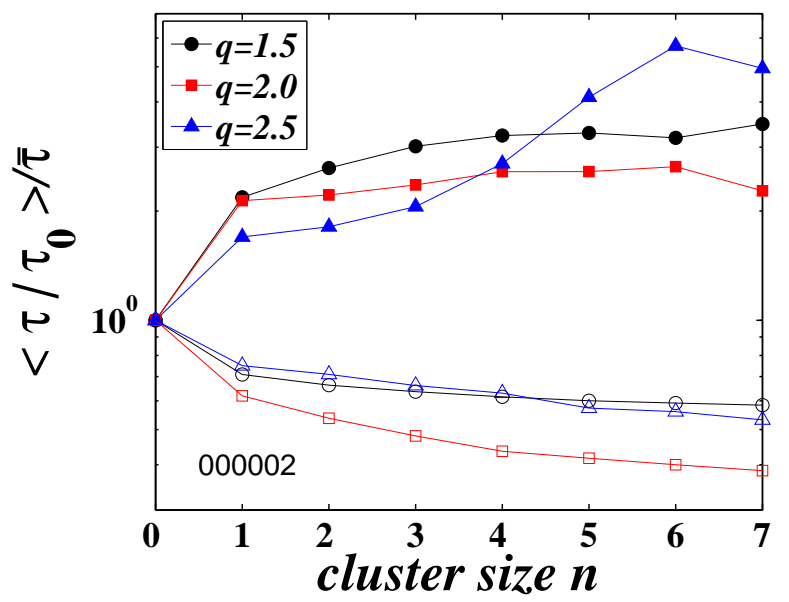

Fig. 7. (Color online) Scaled mean return interval $\left\langle\tau \mid \tau_{0}\right\rangle / \bar{\tau}$ after "+" cluster (filled symbols) and "-" cluster (open symbols) for a representative stock 000002.

To further study the long-term memory of return intervals, we investigate the mean return interval $\left\langle\tau \mid \tau_{0}\right\rangle$ after a cluster of $n$ intervals that are all in a bin $\tau_{0}$. The entire interval sequences are partitioned into two bins, separated by the median value of return intervals. Thus we investigate the mean return interval after a cluster of $n$ intervals consecutively above and below 

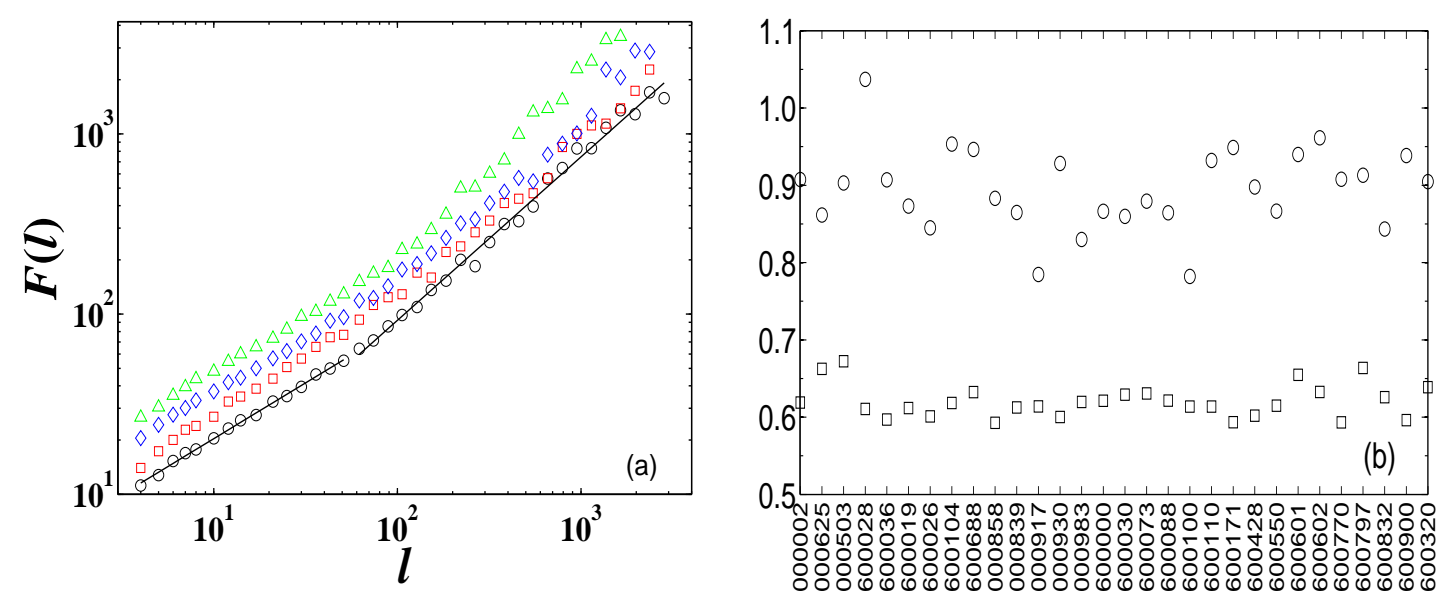

Fig. 8. (a) Detrended fluctuation $F(l)$ of return interval $\tau_{2}$ for four representative stocks. The curves are vertically shifted for clarity. (b) Extracted Hurst exponents $H$ for large scales (circles) and small scales (squares) for the 30 stocks.

the median value, denoted by "+" and "-" clusters. Figure 7 illustrates $\left\langle\tau \mid \tau_{0}\right\rangle$ with respect to the cluster size $n$ for a representative stock 000002 . The results for other stocks are similar. In general, $\left\langle\tau \mid \tau_{0}\right\rangle$ after "+" cluster increases, while $\left\langle\tau \mid \tau_{0}\right\rangle$ after "-" cluster decreases as the cluster size $n$ increases. In other words, a large mean return interval is likely followed by large consecutive return intervals and a small return interval is likely followed by small consecutive return intervals. This observation shows the dependence of the return interval on its previous $n$ consecutive return intervals.

The detrended fluctuation analysis (DFA) method is applied to directly investigate the long-term correlation of return intervals. After removing a linear trend, the DFA method computes the detrended fluctuation $F(l)$ of the time series within a window of $l$ points and determines the exponent $H$ from the scaling form $F(l) \sim l^{H}$, where $H$ is the Hurst exponent [38,39]. For $H>0.5$ the time series is long-term correlated, and for $H=0.5$ the time series is uncorrelated. The detrended fluctuation function $F(l)$ of four representative stocks are shown in Figure 8 a). A crossover behavior is observed: $F(l)$ for small scales $l<50$ obeys a power law with an relatively small exponent and $F(l)$ for large scales $l>50$ obeys a power law with a relatively large exponent. This phenomenon is also observed for other stocks, which is in line with other stock markets [25]. Figure 8 b) illustrates the estimated Hurst exponents $H$ of the 30 stocks. In both regions for small scales and large scales, the Hurst exponents of all the individual stocks are apparently greater than 0.5 , which indicates the existence of longterm memory in return intervals. For shuffled data, the Hurst exponent displays a value close to 0.5 . This confirms that the long-term memory may arise from the long-term memory of volatility records.

\section{Summary and conclusions}

In summary, we have studied the distribution and memory effect of volatility return intervals for 30 most actively traded stocks on the Shanghai and Shenzhen Stock Exchanges. The Kolmogorov-Smirnov tests are performed to examine the scaling behavior of the return interval distributions as well as the particular form of the scaling function. We find that 12 stocks exhibit scaling behaviors in the volatility return interval distributions. We further find that the scaling form of 6 stocks having the best scaling can be excellently approximated by a stretched exponential function $f(x) \sim e^{-\alpha x^{\gamma}}$, and the mean value of the correlation exponent $\gamma$ is estimated to be around 0.31 .

The memory effect is also observed in the volatility return intervals of the 30 stocks. We first calculate the conditional PDF $P_{q}\left(\tau \mid \tau_{0}\right)$ and mean conditional return interval $\left\langle\tau \mid \tau_{0}\right\rangle$, and find that the interval $\tau$ is dependent of its closest previous interval $\tau_{0}$, which indicates the short-term memory of return intervals. We further investigate $\left\langle\tau \mid \tau_{0}\right\rangle$ after a cluster of $n$ intervals consecutively above and below the median value, and find that memory exists in a cluster of long return intervals. In addition, detrended fluctuation analysis of the return intervals shows that the return intervals have long-term memory.

In a nutshell, our work focuses on the study of the statistical properties of volatility return intervals of Chinese stocks. Our main finding is that some stocks show scaling behaviors while the others do not. This novel feature shows the complexity of the Chinese stock market and help us better understand the properties of volatility return intervals.

We thank Z.-Q. Jiang, G.-F. Gu, G.-H. Mu and T. Qiu for helpful discussions and suggestions. This work was partially supported by the Shanghai Educational Development Foundation (No. 2008CG37), the National Natural Science Foundation of China (No. 70501011), the Fok Ying Tong Education Foundation (No. 101086), and the Program for New Century Excellent Talents in University (No. NCET07-0288).

\section{References}

1. R.N. Mantegna, H.E. Stanley, An Introduction to Econophysics: Correlations and Complexity in Finance (Cambridge University Press, Cambridge, 2000)

2. B. Zheng, Mod. Phys. Lett. B 16, 775 (2002)

3. F. Ren, B. Zheng, Phys. Lett. A 313, 312 (2003)

4. F. Ren, B. Zheng, H. Lin, L. Wen, S. Trimper, Physica A 350, 439 (2005) 
5. I. Simonsen, M.H. Jensen, A. Johansen, Eur. Phys. J. B 27, 583 (2002)

6. M.H. Jensen, A. Johansen, I. Simonsen, Int. J. Modern Phys. B 17, 4003 (2003)

7. M.H. Jensen, A. Johansen, I. Simonsen, Physica A 324, 338 (2003)

8. M. Załuska-Kotur, K. Karpio, A. Orłowski, Acta Physica Polonica B 37, 3187 (2006)

9. K. Karpio, M.A. Załuska-Kotur, A. Orłowski, Physica A 375, 599 (2007)

10. W.-X. Zhou, W.-K. Yuan, Physica A 353, 433 (2005)

11. E. Scalas, R. Gorenflo, H. Luckock, F. Mainardi, M. Mantelli, M. Raberto, Quant. Financ. 4, 695 (2004)

12. P.C. Ivanov, A. Yuen, B. Podobnik, Y.K. Lee, Phys. Rev. E 69, 056107 (2004)

13. N. Sazuka, Physica A 376, 500 (2007)

14. Z.-Q. Jiang, W. Chen, W.-X. Zhou, Physica A 387, in press (2008)

15. A. Bunde, J.F. Eichner, S. Havlin, J.W. Kantelhardt, Physica A 330, 1 (2003)

16. A. Bunde, J.F. Eichner, S. Havlin, J.W. Kantelhardt, Physica A 342, 308 (2004)

17. A. Bunde, J.F. Eichner, J.W. Kantelhardt, S. Havlin, Phys. Rev. Lett. 94, 048701 (2005)

18. A. Saichev, D. Sornette, Phys. Rev. Lett. 97, 078501 (2006)

19. C. Pennetta, Eur. Phys. J. B 50, 95 (2006)

20. P. Olla, Phys. Rev. E 76, 011122 (2007)

21. J.F. Eichner, J.W. Kantelhardt, A. Bunde, S. Havlin, Phys. Rev. E 73, 016130 (2006)

22. J.F. Eichner, J.W. Kantelhardt, A. Bunde, S. Havlin, Phys. Rev. E 75, 011128 (2007)

23. M.I. Bogachev, J.F. Eichner, A. Bunde, Phys. Rev. Lett. 99, 240601 (2007)

24. K. Yamasaki, L. Muchnik, S. Havlin, A. Bunde, H.E. Stanley, Proc. Natl. Acad. Sci. USA 102, 9424 (2005)

25. F.-Z. Wang, K. Yamasaki, S. Havlin, H.E. Stanley, Phys. Rev. E 73, 026117 (2006)

26. F. Wang, P. Weber, K. Yamasaki, H. S., H.E. Stanley, Eur. Phys. J. B 55, 123C133 (2007)

27. I. Vodenska-Chitkushev, F.-Z. Wang, P. Weber, K. Yamasaki, S. Havlin, H.E. Stanley, Eur. Phys. J. B 61, 217 (2008)

28. W.S. Jung, F.-Z. Wang, S. Havlin, T. Kaizoji, H.T. Moon, H.E. Stanley, Eur. Phys. J. B 62, 113 (2008)

29. T. Qiu, L. Guo, G. Chen (2008), e-print arXiv:physics/08052194v1

30. F.-Z. Wang, K. Yamasaki, S. Havlin, S.H. E., Phys. Rev. E 77, 016109 (2008)

31. J.W. Lee, K.E. Lee, P.A. Rikvold, J. Korean Phys. Soc. 48, S123 (2006)

32. Z.-Q. Jiang, L. Guo, W.-X. Zhou, Eur. Phys. J. B 57, 347 (2007)

33. X.-H. Ni, W.-X. Zhou (2007), arXiv:0710.2402

34. G.-F. Gu, W. Chen, W.-X. Zhou, Physica A 387, 495 (2008)

35. A. Clauset, C.R. Shalizi, M.E.J. Newman (2007), arXiv:0706.1062

36. M.C. Gonzalez, C.A. Hidalgo, A.-L. Barabasi, Nature 453, 779 (2008)

37. T. Qiu, B. Zheng, F. Ren, S. Trimper, Physica A 378, 387 (2007)

38. C.-K. Peng, S.V. Buldyrev, S. Havlin, M. Simons, H.E. Stanley, A.L. Goldberger, Phys. Rev. E 49, 1685 (1994)

39. J.W. Kantelhardt, E. Koscielny-Bunde, H.H.A. Rego, S. Havlin, A. Bunde, Physica A 295, 441 (2001) 